\title{
Nomogram predict relapse-free survival of patients with thymic epithelial tumors after surgery
}

Yang-Yu Huang ${ }^{1+}$, Lei-Lei $\mathrm{Wu}^{2+}$, Xuan Liu ${ }^{1+}$, Shen-Hua Liang ${ }^{1}$ and Guo-Wei Ma1*

\begin{abstract}
Background: Hematological indicators and clinical characteristics play an important role in the evaluation of the progression and prognosis of thymic epithelial tumors. Therefore, we aimed to combine these potential indicators to establish a prognostic nomogram to determine the relapse-free survival (RFS) of patients with thymic epithelial tumors undergoing thymectomy.

Methods: This retrospective study was conducted on 156 patients who underwent thymectomy between May 2004 and August 2015. Cox regression analysis were performed to determine the potential indicators related to prognosis and combine these indicators to create a nomogram for visual prediction. The prognostic predictive ability of the nomogram was evaluated using the consistency index (C-index), receiver operating characteristic (ROC) curve, and risk stratification. Decision curve analysis was used to evaluate the net benefits of the model.

Results: Preoperative albumin levels, neutrophil-to-lymphocyte ratio (NLR), T stage, and WHO histologic types were included in the nomogram. In the training cohort, the nomogram showed well prognostic ability (C index: 0.902). Calibration curves for the relapse-free survival (RFS) were in good agreement with the standard lines in training and validation cohorts.

Conclusions: Combining clinical and hematologic factors, the nomogram performed well in predicting the prognosis and the relapse-free survival of this patient population. And it has potential to identify high-risk patients at an early stage. This is a relatively novel approach for the prediction of RFS in this patient population.
\end{abstract}

Keywords: Thymic epithelial tumor, Overall survival, Nomogram, Albumin, Ratio of neutrophils to lymphocytes

\section{Background}

Thymic epithelial tumors common occur in the anterior mediastinum and can be divided into thymoma and thymic carcinoma according to histology $[1,2]$. The Masaoka-Koga staging system, which is based on the progression of the primary tumor and the degree of involvement of the surrounding organs, has been widely accepted

\footnotetext{
* Correspondence: magw@sysucc.org.cn

†Yang-Yu Huang, Lei-Lei Wu and Xuan Liu contributed equally to this work. 'State Key Laboratory of Oncology in South China, Sun Yat-sen University Cancer Center, Collaborative Innovation Center for Cancer Medicine, Guangzhou, China

Full list of author information is available at the end of the article
}

for thymoma and thymic carcinoma [3-5]. However, Yanagiya et al. found that age and histological type were significant prognostic factors in their cohort, which were not observed or reported in the Masaoka-Koga staging system [6]. Similarly, the results of the study published by Fukui et al. revealed that the new classification showed a better prognostic effect for thymic tumors than the Masaoka-Koga classification [7, 8]. Moreover, compared with the staging systems for most other malignant tumors, the Masaoka-Koga system does not include the effect of lymph node or distant organ metastasis on prognosis as finely as the TNM staging. 
At the same time, an increasing number of studies have used clinical factors such as history of hypertension, diabetes, [9] smoking, [10] and body mass index (BMI) [11] and hematological indicators including hemoglobin $(\mathrm{Hb})$ [12], neutrophil-to-lymphocyte ratio (NLR) [13, 14], albumin (ALB) [15], and other such indicators to analyze the prognosis of various tumors. However, only few studies have comprehensively analyzed a combination of the two types of indicators to establish a prognostic model for patients with thymic epithelial tumors after thymectomy. Currently, nomograms have been developed for most cancer types [16-18]. Compared with the traditional staging system for many cancers, the use of a nomogram has advantages in terms of prognostic prediction. Therefore, it has been proposed as an alternative method for cancer staging [19-21].

Therefore, in this study, we aimed to use both preoperative hematological indicators and clinical factors to construct a prognostic predictive nomogram for patients with thymic epithelial tumors after thymectomy for a comprehensive evaluation. In addition, the nomogram score was used for risk stratification to identify high-risk patients.

\section{Materials and methods}

\section{Study population}

This study was approved by the Medical Ethics Committee of Sun Yat-sen University Cancer Center (SYSUCC; Approval No. B2020-353-01) and complies with the Declaration of Helsinki.

This study retrospectively analyzed 156 patients who underwent R0 resection of thymus epithelial tumor at
SYSUCC between May 2004 and August 2015. Most patients were included in the training group $(n=116)$, and the remaining patients were included in the verification group $(n=40)$. The inclusion criteria were as follows: (1) patients older than 18 years; (2) patients who underwent thymectomy at our center; (3) presence of histopathologically confirmed thymic epithelial tumors; (4) related laboratory examinations (blood routine, biochemical routine and so on) were completed within 7 days before the operation. 5) the scope of surgical resection was R0 resection. The exclusion criteria and the screening process are shown in Fig. 1.

\section{Clinical data collection}

Data were collected for the following clinical variables: hematological indicators (obtained within 1 week before the operation), neutrophil count (NE), lymphocyte count (LY), platelet count, albumin levels, globulin levels as well as patients' sex, age, smoking history, drinking history (Drinking alcohol every day, and the specific amount of drinking is not limited and described), family history of tumor, underlying disease (hypertension and/ or diabetes), tumor size, histological subtype, myasthenia gravis symptoms, tumor capsule status (complete or incomplete), great vessel infiltration, Masaoka-Koga staging, and $\mathrm{T}$ staging. In addition, $\mathrm{T}$ staging and Masaoka staging were obtained by combining imaging data with intraoperative records and postoperative pathological information, we staged all patients according to the eighth edition of the TNM staging system and the modified Masaoka-Koga staging system.

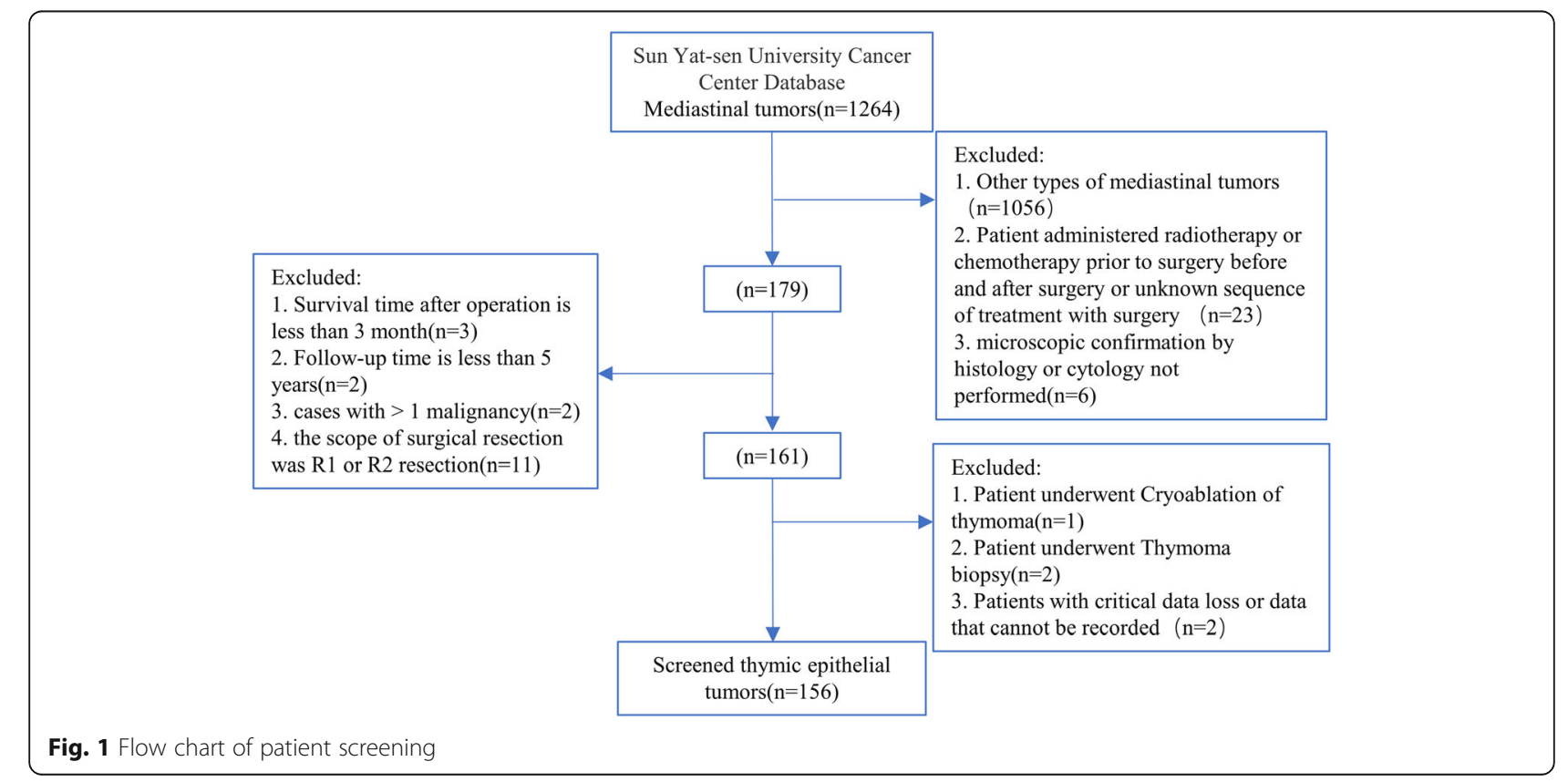




\section{Follow-up}

We followed up patients regularly. In the first 2 years, all patients were followed up every 6-12 months, every 12 months from the third to the fifth year, and then an annual follow-up was continued. The last follow-up date was August 22, 2020. The methods of follow-up were telephone and outpatient. The primary observational endpoint was RFS. RFS was defined as the length of time after primary treatment for a cancer ends that the patient survives without any signs or symptoms of that cancer.

\section{Statistical analysis}

Statistical analysis was performed using SPSS 25.0 (IBM, Chicago, Illinois, USA) and $\mathrm{R}$ software (version 4.0.3; https://www.r-project.org/). In addition to age and tumor size, each component was converted to binary according to the best cutoff value (using X-tile software; http://www.tissuearray.org/rimmlab) according to the best cutoff value defined by the minimum $P$ value method variables. This method has also been applied in other studies [22]. In the training cohort, the Cox regression model was used to analyze risk factors through univariate and multivariate analyses. Univariate analysis was performed to determine important risk factors for RFS. Variables with $P$ values of less than 0.05 were further included in the multivariate Cox proportional hazard regression model. In the final multivariate analysis, $\mathrm{P}$ values of less than 0.05 were considered independent prognostic factors. Then, based on the results of the multivariate Cox analysis, we constructed a nomogram showing three-year and five-year relapse-free survival rates.

The R statistical software packages "rms," "survival," "foreign," "survivalROC," and "rmda" were used to calculate the $C$ index; to generate the calibration curve, receiver operating characteristic (ROC) curves, decision curve analysis (DCA) curve, and Kaplan-Meier (KM) curve; and to construct a nomogram. The nomogram was used to calculate the prognostic risk score for each patient; X-tile was used to divide the patient's score into different risk levels (low risk, medium risk, and high risk) and to show their stratification effect through the KM curve [23]. The C-index, DCA curve, and ROC curve were used to evaluate the predictive ability of the nomogram. All statistical tests were two-sided, and $P$ values of less than 0.05 were considered statistically significant.

\section{Results}

Basic characteristics

A total of 156 patients participated in the study. Among them, 116 patients (Approximate 70\%) were randomly assigned to the training group to build a nomogram, and the remaining 40 patients (Approximate 30\%) were assigned to the verification group. Table 1 shows the data of the clinicopathological characteristics of the 156 patients. The three-year and five-year relapse-free survival rates were 0.932 and 0.905 , respectively. We found that 129 patients $(81.1 \%)$ achieved a five-year relapsefree survival time in all patients. These clinicopathological factors did not differ significantly between the training and validation cohorts.

\section{Univariable and multivariable analyses in the training cohort}

According to the results of univariate Cox regression analysis, there were nine variables related to RFS: WHO histologic types, $\mathrm{T}$ stage, Tumor capsule status, Invasion of great vessels, ALB, Neutrophils (NE), NLR, PLR and systemic immune-inflammation Index (SII) (Table 2). In the multivariate Cox regression analysis, four parameters were defined as independent prognostic factors of RFS: $\mathrm{T}$ stage ( $\mathrm{T} 1$ vs. $\mathrm{T} 2-3$, hazard ratio, $\mathrm{HR}=7.518,95 \%$ confidence interval, CI [1.355-41.718], ALB $(\mathrm{HR}=0.157$, 95\% CI [0.035-0.697]), WHO histologic types (A-AB vs $\mathrm{B} 1-\mathrm{B} 3, \mathrm{HR}=0.379,95 \% \mathrm{CI}[0.070-2.040], \mathrm{A}-\mathrm{AB}$ vs $\mathrm{C}$, $\mathrm{HR}=5.892,95 \% \mathrm{CI}[0.660-52.582])$, and NLR $(\mathrm{HR}=$ $15.426,95 \%$ CI [1.759-135.300]) (Table 2).

\section{Establishment of the nomogram}

According to the results of the multivariate Cox regression analysis, T stage, ALB, WHO histologic types, and NLR were defined as independent prognostic factors, and these factors were integrated to form a nomogram (Fig. 2). In the training cohort, the $\mathrm{C}$ index was 0.902 (95\% CI: 0.843-0.961). Internal calibration curves for the three- and five-year RFS closely matched those of the baseline in the training cohort (Fig. 3Aand B).

\section{Verification of the nomogram}

To better verify the actual predictive power of the nomogram, the above results were verified using the verification group data, showing that the $C$ index was 0.785 (95\% CI: 0.614-0.957), and the five-year and three-year external calibration curves met those of the standard baseline (Fig. 3C and D). We also used the ROC curve to verify the nomogram performance (Fig. 4). The AUC values of the training and validation groups at 3 and 5 years were both greater than 0.65 and by comparing the AUC values of the two groups, the nomogram model were showing well accuracy of the nomogram in predicting RFS.

\section{Decision curve analysis}

Decision Curve Analysis (DCA) is a novel method for evaluating prognostic strategies that can evaluate the predictive power of prognostic models. Figure 5 shows 
Table 1 Patient, tumor, and treatment-related characteristics of thymic tumor $(n=156)$

\begin{tabular}{|c|c|c|c|c|}
\hline \multirow[t]{2}{*}{ Characteristic } & \multicolumn{2}{|c|}{ Training Cohort $(n=116)$} & \multicolumn{2}{|c|}{ Validation Cohort $(n=40)$} \\
\hline & $\mathrm{N}$ & $\%$ & $\mathbf{N}$ & $\%$ \\
\hline \multicolumn{5}{|l|}{ Gender } \\
\hline Male & 63 & 54.3 & 20 & 50.0 \\
\hline Female & 53 & 45.7 & 20 & 50.0 \\
\hline \multicolumn{5}{|l|}{ Age (years) } \\
\hline$\leq 60$ & 89 & 76.7 & 32 & 80.0 \\
\hline$>60$ & 27 & 23.3 & 8 & 20.0 \\
\hline \multicolumn{5}{|l|}{ Smoking history } \\
\hline Never & 84 & 72.4 & 34 & 85.0 \\
\hline Ever & 32 & 27.6 & 6 & 15.0 \\
\hline \multicolumn{5}{|l|}{ Drinking history } \\
\hline No & 100 & 86.2 & 37 & 92.5 \\
\hline Yes & 16 & 13.8 & 3 & 7.5 \\
\hline \multicolumn{5}{|c|}{ Family history of tumor } \\
\hline No & 98 & 84.5 & 34 & 85.0 \\
\hline Yes & 18 & 15.5 & 6 & 15.0 \\
\hline \multicolumn{5}{|c|}{ Underlying diseases } \\
\hline No & 82 & 70.7 & 34 & 85.0 \\
\hline Yes & 34 & 29.3 & 6 & 15.0 \\
\hline \multicolumn{5}{|l|}{ Tumor size $(\mathrm{cm})$} \\
\hline$\leq 6$ & 65 & 56.0 & 24 & 60.0 \\
\hline$>6$ & 51 & 44.0 & 16 & 40.0 \\
\hline \multicolumn{5}{|l|}{ pT stage } \\
\hline $\mathrm{T} 1$ & 91 & 78.4 & 31 & 77.5 \\
\hline $\mathrm{T} 2-3$ & 25 & 21.6 & 9 & 22.5 \\
\hline \multicolumn{5}{|l|}{ Masaoka stage } \\
\hline I & 61 & 52.6 & 15 & 37.5 \\
\hline$\|-1\|$ & 55 & 47.4 & 25 & 62.5 \\
\hline \multicolumn{5}{|l|}{ WHO stage } \\
\hline$A-A B$ & 45 & 38.8 & 18 & 45.0 \\
\hline B1-B3 & 60 & 51.7 & 17 & 42.5 \\
\hline C & 11 & 9.5 & 5 & 12.5 \\
\hline \multicolumn{5}{|c|}{ Myasthenia gravis, } \\
\hline No & 107 & 92.2 & 37 & 92.5 \\
\hline Yes & 9 & 7.8 & 3 & 7.5 \\
\hline \multicolumn{5}{|c|}{ tumor capsule status } \\
\hline Incomplete & 38 & 32.8 & 9 & 22.5 \\
\hline Complete & 78 & 67.2 & 31 & 77.5 \\
\hline \multicolumn{5}{|c|}{ Invasion of great vessels } \\
\hline No & 91 & 78.4 & 33 & 82.5 \\
\hline Yes & 25 & 21.6 & 7 & 17.5 \\
\hline \multicolumn{5}{|l|}{ ALB } \\
\hline$\leq 40.8$ & 29 & 25.0 & 7 & 17.5 \\
\hline$>40.8$ & 87 & 75.0 & 33 & 82.5 \\
\hline
\end{tabular}


Table 1 Patient, tumor, and treatment-related characteristics of thymic tumor $(n=156)$ (Continued)

\begin{tabular}{|c|c|c|c|c|}
\hline \multirow[t]{2}{*}{ Characteristic } & \multicolumn{2}{|c|}{ Training Cohort $(n=116)$} & \multicolumn{2}{|c|}{ Validation Cohort $(n=40)$} \\
\hline & $\mathrm{N}$ & $\%$ & $\mathrm{~N}$ & $\%$ \\
\hline \multicolumn{5}{|l|}{ GLB } \\
\hline$\leq 28.8$ & 58 & 50.0 & 6 & 15.0 \\
\hline$>28.8$ & 58 & 50.0 & 34 & 85.0 \\
\hline \multicolumn{5}{|l|}{$A / G$} \\
\hline$\leq 1.5$ & 54 & 46.6 & 18 & 45.0 \\
\hline$>1.5$ & 62 & 53.4 & 22 & 55.0 \\
\hline \multicolumn{5}{|l|}{$\mathrm{NE}$} \\
\hline$\leq 5.6$ & 102 & 87.9 & 32 & 80.0 \\
\hline$>5.6$ & 14 & 12.1 & 8 & 20.0 \\
\hline \multicolumn{5}{|l|}{ LY } \\
\hline$\leq 2.5$ & 28 & 24.1 & 8 & 20.0 \\
\hline$>2.5$ & 88 & 75.9 & 32 & 80.0 \\
\hline \multicolumn{5}{|l|}{ NE/LY (NLR) } \\
\hline$\leq 3.0$ & 100 & 86.2 & 32 & 80.0 \\
\hline$>3.0$ & 16 & 13.8 & 8 & 20.0 \\
\hline \multicolumn{5}{|l|}{ PLT } \\
\hline$\leq 237$ & 64 & 55.2 & 16 & 40.0 \\
\hline$>237$ & 52 & 44.8 & 24 & 60.0 \\
\hline \multicolumn{5}{|l|}{ PLT/LY (PLR) } \\
\hline$\leq 161.6$ & 104 & 89.7 & 32 & 80.0 \\
\hline$>161.6$ & 12 & 10.3 & 8 & 20.0 \\
\hline \multicolumn{5}{|l|}{ PLT/NE*LY (SII) } \\
\hline$\leq 764.3$ & 102 & 87.9 & 30 & 75.0 \\
\hline$>764.3$ & 14 & 12.1 & 10 & 25.0 \\
\hline
\end{tabular}

NLR neutrophil-to-lymphocyte ratio, $H b$ hemoglobin, $A L B$ albumin, $B M I$ body mass index, $N E$ neutrophil count, $L Y$ lymphocyte count, GLB globulin, SIl systemic immune-inflammation Index, $P L T$ platelet, $P L R$ platelet-lymphocyte ratio, $p T$ stage pathological T stage

the DCA curve of nomogram in the training and validation cohorts. The DCA of the nomogram has a high net benefit, which indicates that the nomogram can have well clinical application.

\section{Risk stratification of OS}

Based on the nomogram scores, patients were divided into low-risk (0-100 points), medium-risk (101-179 points), and high-risk (180 points or higher) subgroups. In the training cohort, there were 77 patients in the lowrisk group, 26 patients in the intermediate-risk group, and 13 patients in the high-risk group. In the validation cohort, 29 patients were included in the low-risk group, 7 patients were included in the medium-risk group, and 4 patients were included in the high-risk group. There were significant differences in the incidence of RFS among the subgroups, and the relapse-free survival rate of the high-risk subgroup was lower than that of the other groups $(P<0.05)$ (Fig. 6).

\section{Discussion}

In this study, we obtained results from 156 patients at SYSUCC, and ALB, NLR, T stage, and WHO histologic types were found to be independent prognostic factors of RFS. We developed a nomogram that can effectively predict the RFS rate of patients with thymic epithelial tumors at 3 and 5 years.

Slow disease progression and good prognosis have largely limited research on thymic epithelial tumors. At the same time, in the era of precision medicine, it is very important to analyze patient information as comprehensively as possible to screen factors affecting the prognosis for treatment decision-making. At present, the MasaokaKoga staging system is still the gold standard for predicting the prognosis of thymic epithelial tumors. However, similar to the results of Fukui et al., [7] our results show that the $\mathrm{T}$ staging is better than the Masaoka-Koga staging in predicting prognosis. Therefore, the nomogram developed in this study was mainly included $\mathrm{T}$ stage. Based on this, the nomogram along with the patient's various 
Table 2 Univariate and multivariate analysis results in Training cohort $(n=116)$

\begin{tabular}{|c|c|c|c|c|}
\hline \multirow[t]{2}{*}{ Variable } & \multirow{2}{*}{$\begin{array}{l}\text { Univariate analysis } \\
\mathrm{P}\end{array}$} & \multicolumn{3}{|l|}{ Multivariate analysis } \\
\hline & & HR & $95 \% \mathrm{Cl}$ & $\mathbf{P}$ \\
\hline $\begin{array}{l}\text { Gender } \\
\text { Male vs Female }\end{array}$ & .545 & & & \\
\hline $\begin{array}{l}\text { Age (years) } \\
\leq 60 \text { vs }>60\end{array}$ & .175 & & & \\
\hline $\begin{array}{l}\text { Smoking history } \\
\text { Never vs Ever }\end{array}$ & .516 & & & \\
\hline $\begin{array}{l}\text { Drinking history } \\
\text { No vs Yes }\end{array}$ & .128 & & & \\
\hline $\begin{array}{l}\text { Family history of tumor } \\
\text { No vs Yes }\end{array}$ & .858 & & & \\
\hline $\begin{array}{l}\text { Underlying diseases } \\
\text { No vs Yes }\end{array}$ & .176 & & & \\
\hline $\begin{array}{l}\text { Tumor size } \\
\leq 6 \text { vs }>6\end{array}$ & .225 & & & \\
\hline pT stage & .020 & Reference & & \\
\hline T1 vs T2-3 & & 7.518 & $1.355-41.718$ & .021 \\
\hline $\begin{array}{l}\text { Masaoka stage } \\
\text { I vs I|-III }\end{array}$ & .097 & & & \\
\hline WHO stage & .015 & Reference & & .016 \\
\hline$A-A B$ vs B1-B3 & & .379 & $.070-2.040$ & \\
\hline$A-A B$ vs $C$ & & 5.892 & $.660-52.582$ & \\
\hline $\begin{array}{l}\text { Myasthenia gravis, } \\
\text { No vs Yes }\end{array}$ & .434 & & & \\
\hline $\begin{array}{l}\text { tumor capsule status } \\
\text { Incomplete vs Complete }\end{array}$ & .000 & & & .088 \\
\hline $\begin{array}{l}\text { Invasion of great vessels } \\
\text { No vs Yes }\end{array}$ & .000 & & & .117 \\
\hline ALB & .001 & Reference & & \\
\hline$\leq 40.8$ vs $>40.8$ & & .157 & $.035-.697$ & .015 \\
\hline $\begin{array}{l}\text { GLB } \\
\leq 28.8 \text { vs }>28.8\end{array}$ & .446 & & & \\
\hline $\begin{array}{l}\mathrm{A} / \mathrm{G} \\
\leq 1.5 \text { vs }>1.5\end{array}$ & .344 & & & \\
\hline $\begin{array}{l}\mathrm{NE} \\
\leq 5.6 \text { vs }>5.6\end{array}$ & .004 & & & .261 \\
\hline $\begin{array}{l}\mathrm{LY} \\
\leq 2.5 \text { VS }>2.5\end{array}$ & .305 & & & \\
\hline NE/LY (NLR) & .000 & Reference & & \\
\hline$\leq 3.0$ vs $>3.0$ & & 15.426 & $1.759-135.300$ & .014 \\
\hline $\begin{array}{l}\text { PLT } \\
\leq 237 \text { vs }>237\end{array}$ & .075 & & & \\
\hline $\begin{array}{l}\text { PLT/LY (PLR) } \\
\leq 161.6 \text { VS }>161.6\end{array}$ & .041 & & & .082 \\
\hline $\begin{array}{l}\text { PLT/NE*LY (SII) } \\
\leq 764.3 \text { VS }>764.3\end{array}$ & .010 & & & .196 \\
\hline
\end{tabular}

NLR neutrophil-to-lymphocyte ratio, $H b$ hemoglobin, $A L B$ albumin, $B M I$ body mass index, $N E$ neutrophil count, $L Y$ lymphocyte count, GLB globulin, SIl systemic immune-inflammation Index, PLT platelet, PLR platelet-lymphocyte ratio, $p T$ stage pathological T stage

clinical and hematological indicators was used to construct a prognostic model. The nomogram has the advantage of a multi-dimensional comprehensive prediction of prognosis [24]. Some studies have reported nomograms related to the prognosis of thymic epithelial tumors. However, they considered only the clinicopathological 


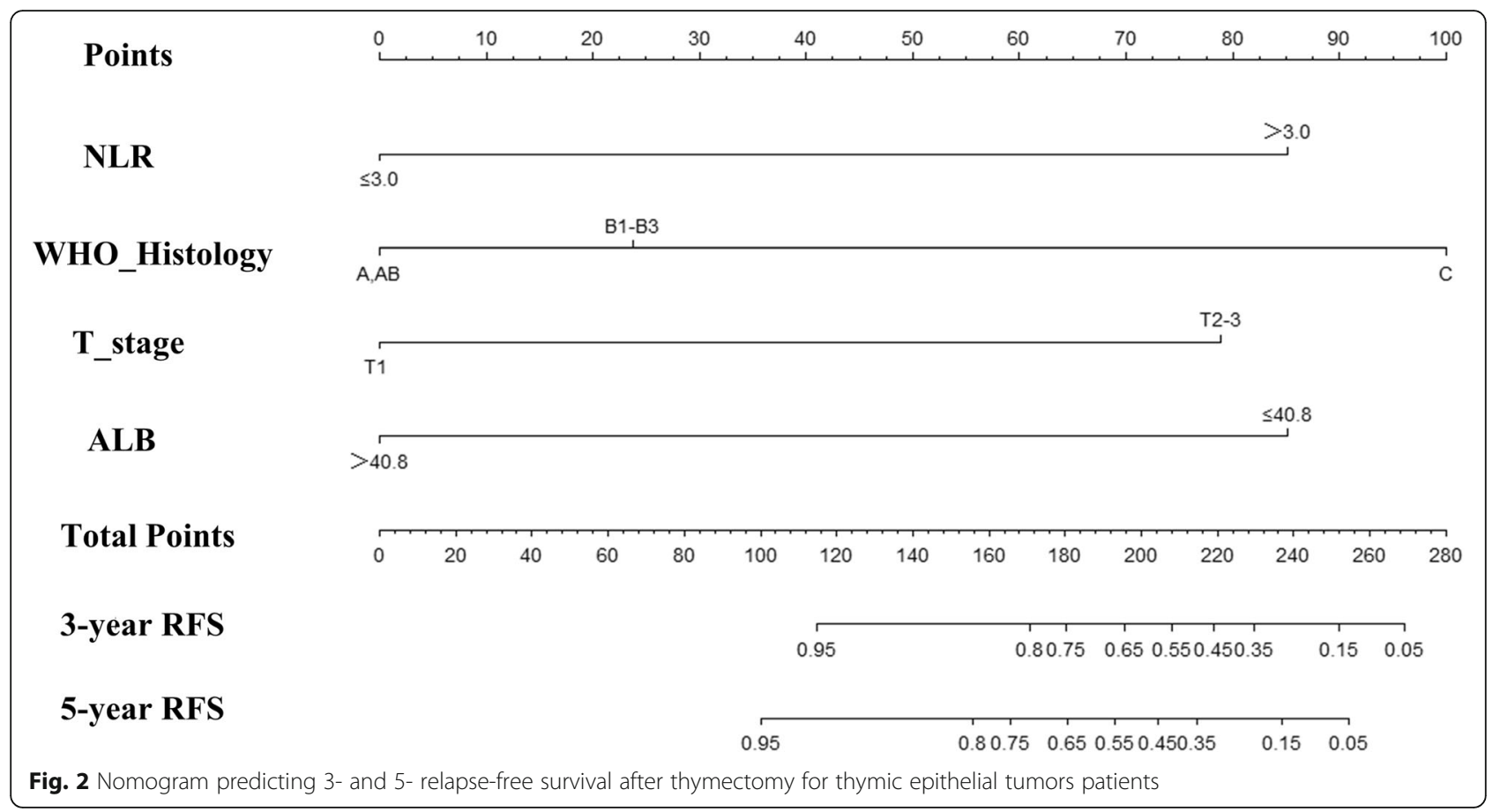

characteristics or treatment methods of patients and do not consider relevant hematological indicators. At the same time, many studies on hematological indicators have shown that $\mathrm{Hb}, \mathrm{NE}, \mathrm{LY}$, platelet (PLT), NLR, ALB, GLB, ALB/GLB, platelet-lymphocyte ratio (PLR), NLR, and SII
$[12,13,15,25]$ have potential to become prognostic hematological indicators for various tumors including thymic epithelial tumors.

Our nomogram is composed of several factors that affect prognosis, which are commonly used in clinical

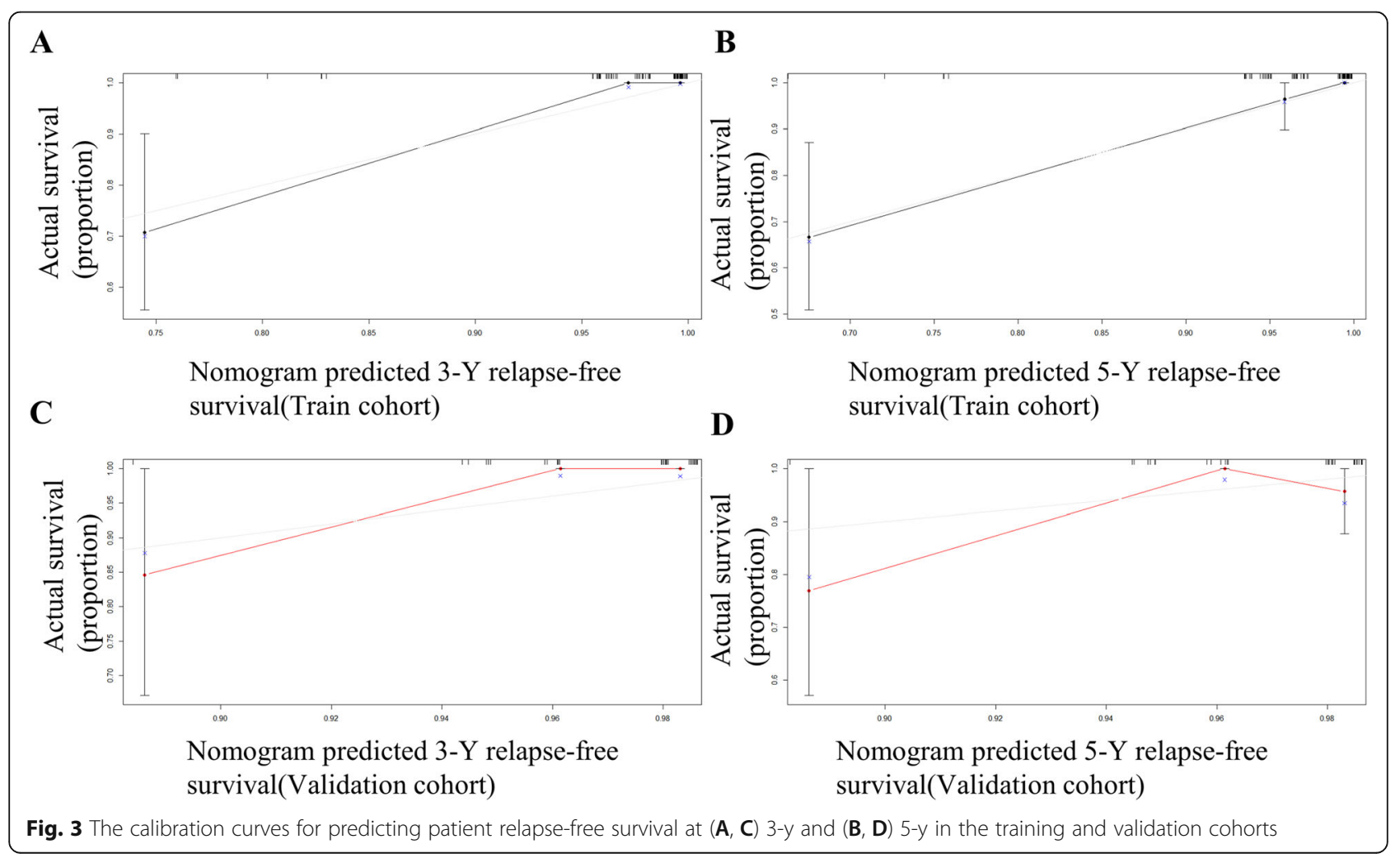



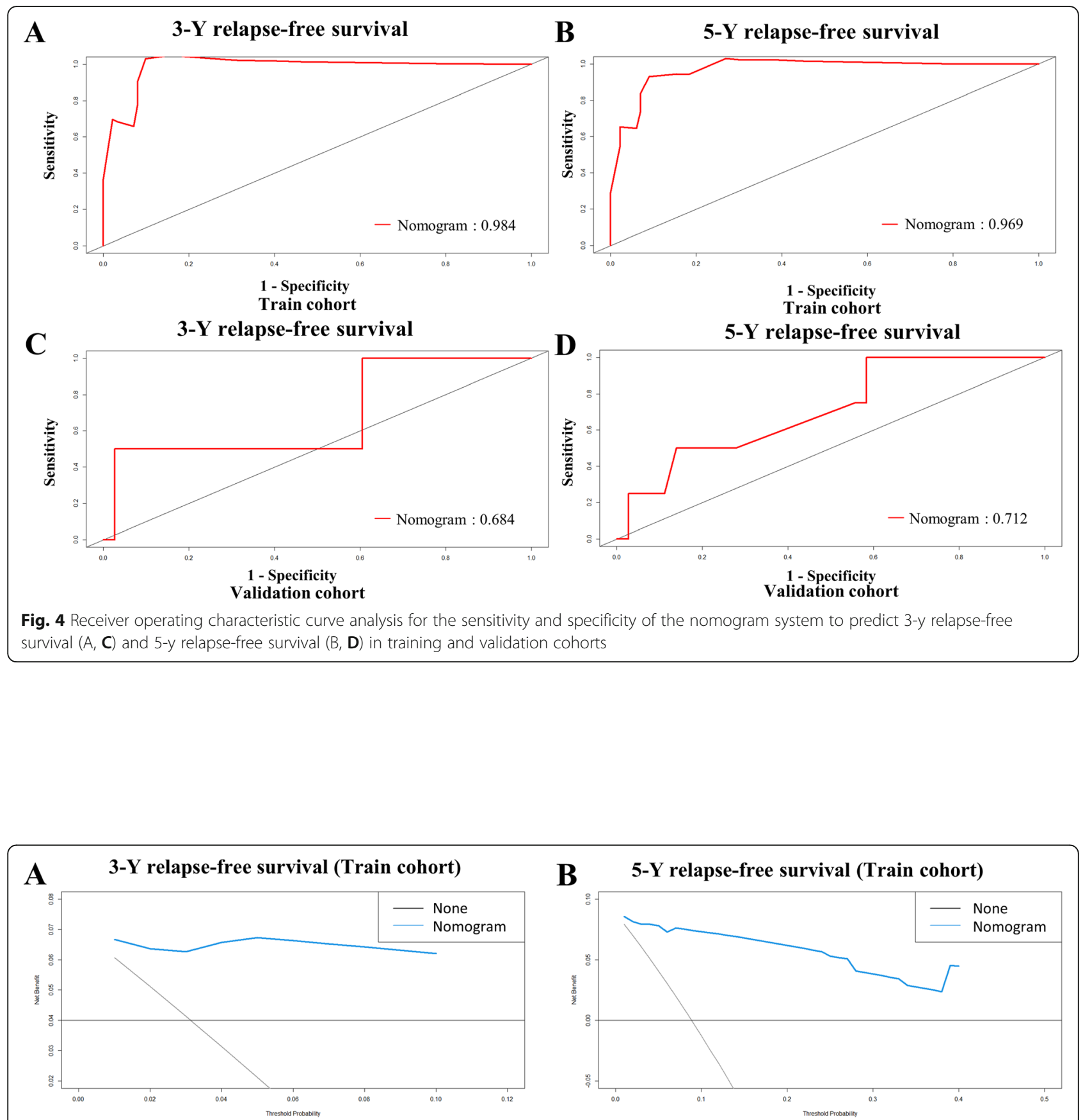

C 3-Y relapse-free survival(Validation cohort)

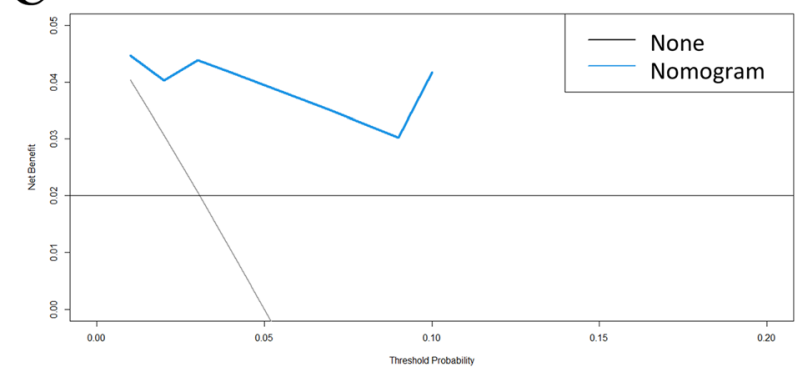

D 5-Y relapse-free survival (Validation cohort)

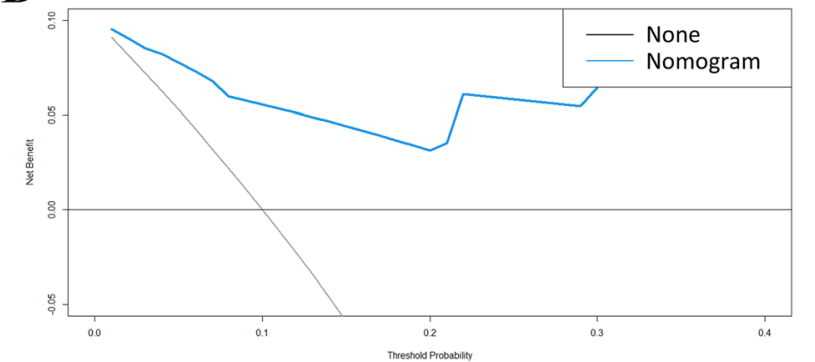

Fig. 5 Decision curve analysis of the training cohort (A, B) and validation cohort (C, D) for 3- and 5-years relapse-free survival 


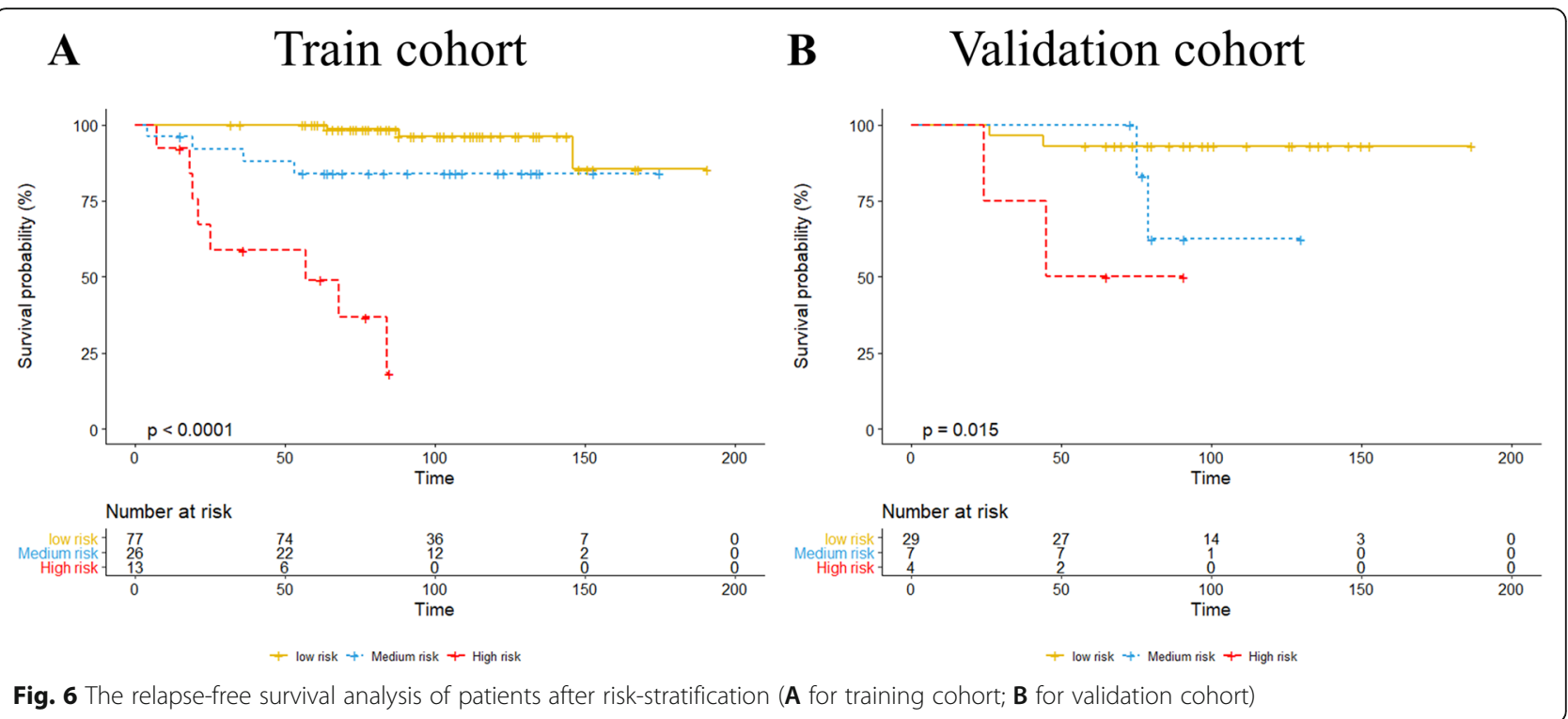

practice. The nomogram showed that higher WHO pathological stage was related to poor RFS in patients with thymic epithelial tumors. This finding is consistent with the findings of other studies [26]. In terms of T staging, lower $\mathrm{T}$ staging can result in a more satisfactory RFS. In the studies of other researchers, the patient's preoperative clinical staging was a factor affecting the prognosis of thymic epithelial tumors [27]. At the same time, we found that elevated NLR was associated with poor prognosis in patients with thymic epithelial tumors. This is the same as other people's research results $[6$, 28]. In addition, a higher preoperative serum ALB level can often result in a more satisfactory prognosis, which is consistent with the findings of other studies reporting better prognosis in patients with thymic epithelial tumors with higher ALB levels [15].

In predicting the prognosis of certain cancers, nomograms have been developed and proven to be more accurate than traditional staging systems [29, 30]. Therefore because thymectomy is an effective treatment for thymic epithelial tumors, [26, 31-33] we constructed a prognostic nomogram for patients with thymic epithelial tumors after surgery. The nomogram performs well in predicting the survival rate. Its prediction is supported by the $C$ index $(0.902$ and 0.785 for the training and verification cohorts, respectively), and the calibration curve was consistent with that of the baseline. The nomogram has high accuracy in predicting survival, and the DCA curve also showed that the nomogram had well predictive ability.

To the best of our knowledge, this is a relatively new attempt to develop a prognostic RFS nomogram for patients with thymic epithelial tumors by combining hematological and clinical indicators. Although this nomogram did not include many hematological indicators in the end, it combines clinical indicators, hematological indicators and other important clinical information to achieve the ultimate goal of integrating multi-dimensional data to jointly predict the prognosis of thymic epithelial tumors.

This study has several limitations. First, this was a retrospective study. Moreover, this was only a singlecenter study that included a small number of patients. Further research including more number of cases is still needed to verify our results. Second, tumor markers (CEA, SCC, AFP, etc.) and other potentially valuable hematological indicators were not included in this study. Third, the dynamic changes in hematological indicators considered in this study were not followed up after the operation.

\section{Conclusions}

In summary, by combining hematological and clinical indicators, we established and validated a nomogram for predicting the relapse-free survival of patients with thymic epithelial tumors. This convenient nomogram had well performance to distinguish the prognosis and risk of patients. Our findings suggest that it may be a potentially easy-to-use tool for physicians and can aid in postoperative personalized prognosis assessment and early identification of high-risk patients. Although the nomogram appears useful for prognostication and identifying high-risk patients, further prospective studies are needed to validate the nomogram and confirm the contribution of each prognostic factor.

\section{Abbreviations}

NLR: Neutrophil-to-lymphocyte ratio; Hb: Hemoglobin; ALB: Albumin; BMI: Body mass index; NE: Neutrophil count; LY: Lymphocyte count; 
GLB: Globulin; SIl: Systemic immune-inflammation Index; PLT: Platelet; PLR: Platelet-lymphocyte ratio; PT stage: Pathological T stage; OS: Overall survival; C-index: Consistency index; ROC: Receiver operating characteristic; DCA: Decision curve analysis; KM: Kaplan-Meier; RFS: Relapse-free survival.

\section{Supplementary Information}

The online version contains supplementary material available at https://doi. org/10.1186/s12885-021-08585-y.

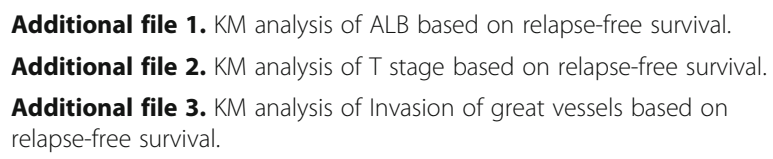

Additional file 4. KM analysis of tumor capsule status based on relapsefree survival.

Additional file 5. KM analysis of SII based on relapse-free survival. Additional file 6. KM analysis of WHO Histology based on relapse-free survival.

Additional file 7. KM analysis of NE based on relapse-free survival.

Additional file 8. KM analysis of PLR based on relapse-free survival.

Additional file 9. KM analysis of NLR based on relapse-free survival.

\section{Acknowledgments}

We would like to thank the Sun Yat-sen University Cancer Center for providing valuable clinical data, Thanks to our respected pioneer, Professor Tie-Hua Rong, who oriented us to surgical oncology. We thank our patients who silently provided data, Finally, we are grateful to Editage (https://app.editage. $\mathrm{com} /$ ) for its excellent polishing work.

\section{Authors' contributions}

Conception and design of the work: MGW, HYY and WLL. Provision of study materials or patients: MGW. Acquisition of data: HYY, WLL and LX. Analysis of data: MGW, HYY and WLL. Interpretation of data: MGW, HYY. HYY and LSH drafted the manuscript; MGW and HYY substantially revised the manuscript. All authors read and approved the final manuscript.

\section{Funding}

This work was supported by grants from the Wu Jie-ping Medical Foundation. (Nos. 320.6750.2020-15-7).

\section{Availability of data and materials}

Data from this study are available to any interested researchers upon reasonable request to the corresponding author.

\section{Declarations}

\section{Ethics approval and consent to participate}

This study was approved by the Medical Ethics Committee of Sun Yat-sen University Cancer Center (SYSUCC; Approval, No. B2020-353-01) and complies with the Declaration of Helsinki. At the same time, this study has obtained the exemption of informed consent application from the Ethics Committee of Sun Yat-sen University Cancer Center.

\section{Consent for publication}

Not applicable.

\section{Competing interests}

The authors disclose no conflicts of interest.

\section{Author details}

${ }^{1}$ State Key Laboratory of Oncology in South China, Sun Yat-sen University Cancer Center, Collaborative Innovation Center for Cancer Medicine, Guangzhou, China. 'Department of Thoracic Surgery, Shanghai Pulmonary Hospital, School of Medicine, Tongji University, Shanghai, P. R. China.
Received: 12 April 2021 Accepted: 12 July 2021

Published online: 22 July 2021

\section{References}

1. Charles R, Thomas CD Jr, et al. Thymoma: State of the Art. J Clin Oncol. 1999;17(7):2280-9.

2. Marx A, Ströbel P, Badve SS, Chalabreysse L, Chan JKC, Chen G, et al. ITMIG consensus statement on the use of the WHO histological classification of Thymoma and Thymic carcinoma: refined definitions, histological criteria, and reporting. J Thorac Oncol. 2014;9(5):596-611. https://doi.org/10.1097/ JTO.0000000000000154.

3. Masaoka A, et al. Staging system of Thymoma. J Thorac Oncol. 2010;5(10): S304-12. https://doi.org/10.1097/JTO.0b013e3181f20c05.

4. Filosso PL, Ruffini E, Lausi PO, Lucchi M, Oliaro A, Detterbeck F. Historical perspectives: the evolution of the thymic epithelial tumors staging system. Lung Cancer. 2014;83(2):126-32. https://doi.org/10.1016/j.lungcan.2013.09. 013.

5. Litvak AM, Woo K, Hayes S, Huang J, Rimner A, Sima CS, et al. Clinical characteristics and outcomes for patients with thymic carcinoma: evaluation of Masaoka staging. J Thorac Oncol. 2014;9(12):1810-5. https://doi.org/10.1 097/JTO.0000000000000363.

6. Yanagiya M, Nitadori Jl, Nagayama K, Anraku M, Sato M, Nakajima J. Prognostic significance of the preoperative neutrophil-to-lymphocyte ratio for complete resection of thymoma. Surg Today. 2018;48(4):422-30. https:// doi.org/10.1007/s00595-017-1602-y.

7. Fukui T, Fukumoto K, Okasaka T, Kawaguchi K, Nakamura S, Hakiri S, et al. Clinical evaluation of a new tumour-node-metastasis staging system for thymic malignancies proposed by the International Association for the Study of Lung Cancer staging and prognostic factors committee and the international Thymic malignancy interest group. Eur J Cardiothorac Surg. 2016;49(2):574-9. https://doi.org/10.1093/ejcts/ezv389.

8. Ried M, Eicher MM, Neu R, Sziklavari Z, Hofmann HS. Evaluation of the new TNM-staging system for thymic malignancies: impact on indication and survival. World J Surg Oncol. 2017;15(1):214. https://doi.org/10.1186/s12957017-1283-4.

9. Heus C, Bakker N, Verduin WM, Doodeman HJ, Houdijk APJ. Impact of body composition on surgical outcome in rectal Cancer patients, a retrospective cohort study. World J Surg. 2019;43(5):1370-6. https://doi.org/10.1007/s002 68-019-04925-z.

10. Yanagiya M, Matsumoto J, Kawahara T, Yamaguchi H, Nagayama K, Anraku $M$, et al. Influence of smoking and histologic subtype on developing Extrathymic malignancy in Thymoma patients. Ann Thorac Surg. 2019; 107(5):1532-9. https://doi.org/10.1016/j.athoracsur.2018.11.038.

11. Renfro LA, Loupakis F, Adams RA, Seymour MT, Heinemann V, Schmoll HJ, et al. Body mass index is prognostic in metastatic colorectal Cancer: pooled analysis of patients from first-line clinical trials in the ARCAD database. J Clin Oncol. 2016;34(2):144-50. https://doi.org/10.1200/JCO.2015.61.6441.

12. Caro JJ, Salas M, Ward A, et al. Anemia as an independant prognostic factor for survival in patients with cancera systematic, quantitative review. Am Cancer Soc. 2001:91:2214-21.

13. De Giorgi U, Procopio G, Giannarelli D, et al. Association of Systemic Inflammation Index and Body Mass Index with survival in patients with renal cell Cancer treated with Nivolumab. Clin Cancer Res. 2019;25(13): 3839-46. https://doi.org/10.1158/1078-0432.CCR-18-3661.

14. Gemenetzis G, Bagante F, Griffin JF, Rezaee N, Javed AA, Manos LL, et al. Neutrophil-to-lymphocyte ratio is a predictive marker for invasive malignancy in Intraductal papillary mucinous neoplasms of the pancreas. Ann Surg. 2017;266(2):339-45. https://doi.org/10.1097/SLA.0000000000001 988.

15. Xin $Y$, Cai $H, W u L$, et al. The effect of Immunonutrition on the postoperative complications in Thymoma with myasthenia gravis. Mediat Inflamm. 2016;2016:8781740.

16. Liu Z, Meng X, Zhang H, Li Z, Liu J, Sun K, et al. Predicting distant metastasis and chemotherapy benefit in locally advanced rectal cancer. Nat Commun. 2020;11(1):4308. https://doi.org/10.1038/s41467-020-18162-9.

17. Tang, Xin-Ran, Li, Ying-Qin,Liang, Shao-Bo., et al., Development and validation of a gene expression-based signature to predict distant metastasis in locoregionally advanced nasopharyngeal carcinoma: a retrospective, multicentre, cohort study. Lancet Oncol, 2018. 19(3): p. 382393, doi: https://doi.org/10.1016/S1470-2045(18)30080-9. 
18. Fakhry C, Zhang Q, Nguyen-Tân PF, et al. Development and Validation of Nomograms Predictive of Overall and Progression-Free Survival in Patients With Oropharyngeal Cancer. J Clin Oncol. 35:4057-65.

19. Diallo, Alhassane, Jacobi, Heike,Cook, Arron., et al., Survival in patients with spinocerebellar ataxia types 1, 2, 3, and 6 (EUROSCA): a longitudinal cohort study. Lancet Neurol, 2018. 17(4): p. 327-334, doi: https://doi.org/10.1016/ S1474-4422(18)30042-5.

20. Hwang, W. L.,Tendulkar, R. D.,Niemierko, A., Agrawal S., Stephans K. L., Spratt D. E., Hearn J. W., Koontz B. F., Lee W. R., Michalski J. M., Pisansky T. M., Liauw S. L., Abramowitz M. C., Pollack A., Moghanaki D., Anscher M. S., den R. B., Zietman A. L., Stephenson A. J., Efstathiou J. A., Comparison between adjuvant and early-salvage Postprostatectomy radiotherapy for prostate Cancer with adverse pathological features. JAMA Oncol, 2018. 4(5): p. e175230, doi: https://doi.org/10.1001/jamaoncol.2017.5230.

21. Olofsson Bagge R, Kicinski M, Faries MB, et al. Factors Affecting Sentinel Node Metastasis in Thin (T1) Cutaneous Melanomas: Development and External Validation of a Predictive Nomogram. J Clin Oncol. 2020;38(14). https://doi.org/10.1200/JCO.19.01902.

22. Wu LL, Liu X, Jiang WM, Huang W, Lin P, Long $H$, et al. Stratification of patients with stage IB NSCLC based on the 8th edition of the American joint committee on Cancer (AJCC) staging manual. Front Oncol. 2020;10: 571. https://doi.org/10.3389/fonc.2020.00571.

23. Zhang LL, Li YY, Hu J, Zhou GQ, Chen L, Li WF, et al. Proposal of a pretreatment nomogram for predicting local recurrence after intensitymodulated radiation therapy in T4 nasopharyngeal carcinoma: a retrospective review of 415 Chinese patients. Cancer Res Treat. 2018;50(4): 1084-95. https://doi.org/10.4143/crt.2017.359.

24. Balachandran, Vinod P.,Gonen, Mithat,Smith, J. Joshua, et al., Nomograms in oncology: more than meets the eye. Lancet Oncol, 2015. 16(4): p. e173e180, doi: https://doi.org/10.1016/S1470-2045(14)71116-7.

25. Ocana A, Nieto-Jimenez C, Pandiella A, et al. Neutrophils in cancer: prognostic role and therapeutic strategies. Mol Cancer. 2017;16(1):137. https://doi.org/10.1186/s12943-017-0707-7.

26. Liu H, ZhiTao G, Qiu B, et al. A Recurrence Predictive Model for Thymic Tumors and Its Implication for Postoperative Management: a Chinese Alliance for Research in Thymomas database study. J Thorac Oncol. 2020; 15(3):448-56.

27. Nicholson AG, Detterbeck FC, Marino M, Kim J, Stratton K, Giroux D, et al. The Thymic epithelial tumors staging project: proposals for the $T$ component for the forthcoming (8th) edition of the TNM classification of malignant tumors. J Thorac Oncol. 2014;9(9):S73-80. https://doi.org/10.1097/ JTO.0000000000000303.

28. Muriana P, Carretta A, Ciriaco P, Bandiera A, Negri G. Assessment of the prognostic role of neutrophil-to-lymphocyte ratio following complete resection of thymoma. J Cardiothorac Surg. 2018;13(1):119. https://doi.org/1 0.1186/s13019-018-0805-7.

29. Sternberg CN. Are nomograms better than currently available stage groupings for bladder cancer? J Clin Oncol. 2006;24(24):3819-20. https://doi. org/10.1200/JCO.2006.07.1290.

30. Dalela D, Santiago-Jiménez M, Yousefi K, et al. Genomic Classifier Augments the Role of Pathological Features in Identifying Optimal Candidates for Adjuvant Radiation Therapy in Patients With Prostate Cancer: Development and Internal Validation of a Multivariable Prognostic Model. J Clin Oncol. 35: 1982-90.

31. Okereke IC, Kesler KA, Morad MH, Mi D, Rieger KM, Birdas TJ, et al. Prognostic indicators after surgery for thymoma. Ann Thorac Surg. 2010; 89(4):1071-7; discussion 1077-9. https://doi.org/10.1016/j.athoracsur.2010.01. 026.

32. Kondo $\mathrm{K}$, Monden $\mathrm{Y}$. Therapy for thymic epithelial tumors: a clinical study of 1,320 patients from Japan. Ann Thorac Surg. 2003;76(3):878-84. https://doi. org/10.1016/50003-4975(03)00555-1.

33. Weksler B, Dhupar R, Parikh V, Nason KS, Pennathur A, Ferson PF. Thymic carcinoma: a multivariate analysis of factors predictive of survival in 290 patients. Ann Thorac Surg. 2013;95(1):299-303. https://doi.org/10.1016/j.a thoracsur.2012.09.006.

\section{Publisher's Note}

Springer Nature remains neutral with regard to jurisdictional claims in published maps and institutional affiliations.

\section{Ready to submit your research? Choose BMC and benefit from:}

- fast, convenient online submission

- thorough peer review by experienced researchers in your field

- rapid publication on acceptance

- support for research data, including large and complex data types

- gold Open Access which fosters wider collaboration and increased citations

- maximum visibility for your research: over $100 \mathrm{M}$ website views per year

At BMC, research is always in progress.

Learn more biomedcentral.com/submissions 